\title{
Resolution Des Problemes Mathematiques En Formation Et Applications
}

\section{Sbaa Mohammed et El aydi M'hamed}

Centre Régional des Métiers de l'éducation et de la Formation Casablanca, Settat, Maroc

\section{Yadir Said}

Laboratoire LEIE, Département de Physique, Faculté des Sciences, UCD, El Jadida, Maroc

\section{Radi Bouchaib}

Laboratoire LIMII, Faculté des Sciences et Techniques, Settat

doi: 10.19044/esj.2017.v13n24p389 URL:http://dx.doi.org/10.19044/esj.2017.v13n24p389

\begin{abstract}
Mathematics occupies a privileged place in the educational system. It is one of the main disciplines in a learner's school life that allows him to develop problem-solving strategies and different learning abilities. Indeed, it is very important to make the learner to become active in the learning process of Mathematics. As a result, problem solving has become a crucial aspect. It helps the learner in the development of strategies for problem solving. Also, the learner must be adaptive to new situations from other disciplines or everyday life. In this study, we used the Georges Polya's "How solve it" guide and we applied it to solving some problems by insisting on the importance of applications in the teaching of Mathematics. We talked about the definition of the open problem and its educational objectives according to the IREM of Lyon. Then we discussed the activities by describing some difficulties encountered by the trainee teachers. These activities include: (1) A problem solved by three different methods: analytical geometry, GeoGebra software, and the Maple software to make a comparison of methods by practice; (2) Solving some open problems. We concluded this study by implementing problem-solving strategies and reasoning methods as tools for trainee teachers for "solving some open problems".
\end{abstract}

Keywords: Mathematical didactics, problem resolution, open problems, Teacher training 


\section{Résumé}

Les mathématiques occupent une place privilégiée dans le système éducatif. Elle est l'une des principales disciplines dans la vie scolaire d'un apprenant et elle permet de développer des stratégies de résolution de problèmes, ainsi que des différentes capacités d'apprentissage. En effet, il est très important de rendre l'apprenant actif dans le processus d'enseignement/ apprentissage des mathématiques. Donc la résolution des problèmes devient un aspect crucial et favorise chez l'apprenant le développement de stratégies de résolution de problèmes et de s'adapter à des situations nouvelles issues des autres disciplines ou de la vie quotidienne. Dans ce travail, nous avons évoqué le guide de Georges Polya «Comment poser et résoudre un problème » et nous l'avons appliqué à la résolution de certains problèmes en insistant sur l'importance des applications dans l'enseignement des mathématiques. Nous avons parlé de la définition du problème ouvert et ses objectifs pédagogiques selon l'IREM de Lyon. Ensuite nous avons traité des activités en décrivant quelques difficultés rencontrées par les professeurs stagiaires. Parmi les activités traitées, nous pouvons citer:

- Un problème résolu par trois méthodes différentes en faisant recours à la géométrie analytique et en utilisant le logiciel Geogebra et le logiciel Maple pour faire une comparaison des méthodes par la pratique.

- La résolution de quelques problèmes ouverts.

Et nous avons conclu le travail par la mise en œuvre des stratégies de résolution de problèmes et des méthodes de raisonnement comme outils pour les professeurs stagiaires pour "la résolution de certains problèmes ouverts".

Mots-clés: Didactiques mathématiques, résolution des problèmes, problèmes ouverts, Formation des enseignants

\section{Introduction}

L'enseignement des mathématiques vise avant tout de développer les capacités de l'apprenant pour résoudre des situations problèmes.

En effet, faire des mathématiques c'est synonyme de résoudre des problèmes. C'est la mise en pratique de différentes stratégies et des connaissances fermement acquises pour aboutir à un résultat; le vérifier et l'interpréter sous diverses formes (tableaux, graphiques etc.).

Il est impératif que l'apprenant soit capable de modéliser un problème, l'identifier et mettre en œuvre une stratégie pour le résoudre (Exemple la stratégie de George Polya).

C'est une discipline qui nous permet de résoudre d'autres problèmes issus d'autres disciplines comme la sociologie, l'épidémiologie, 
l'astronomie, la physique ou tout simplement des problèmes rencontrés dans la vie quotidienne. Bref, l'apprentissage des mathématiques en utilisant des situations-problèmes nous permet de réfléchir, analyser et résoudre des problèmes d'une manière plus efficace.

\section{Résolution des Problèmes Mathématiques \\ Guide de Polya Pour Résoudre un Problème}

Savoir résoudre un problème, cela s'apprend ! C'était le credo du mathématicien hongrois George Polya quand il publia en 1957 son livre « How to solve it », en Français « Comment le résoudre ». Traduit dans plus de 17 langues et vendu à l'époque à plus d'un million d'exemplaires, le livre de George Polya est vite devenu la bible des étudiants en science. Brillant pédagogue, Polya avait identifié les quatre principes élémentaires à respecter pour se donner un maximum de chances de résoudre un problème posé, nous donnons des points de chaque étape:

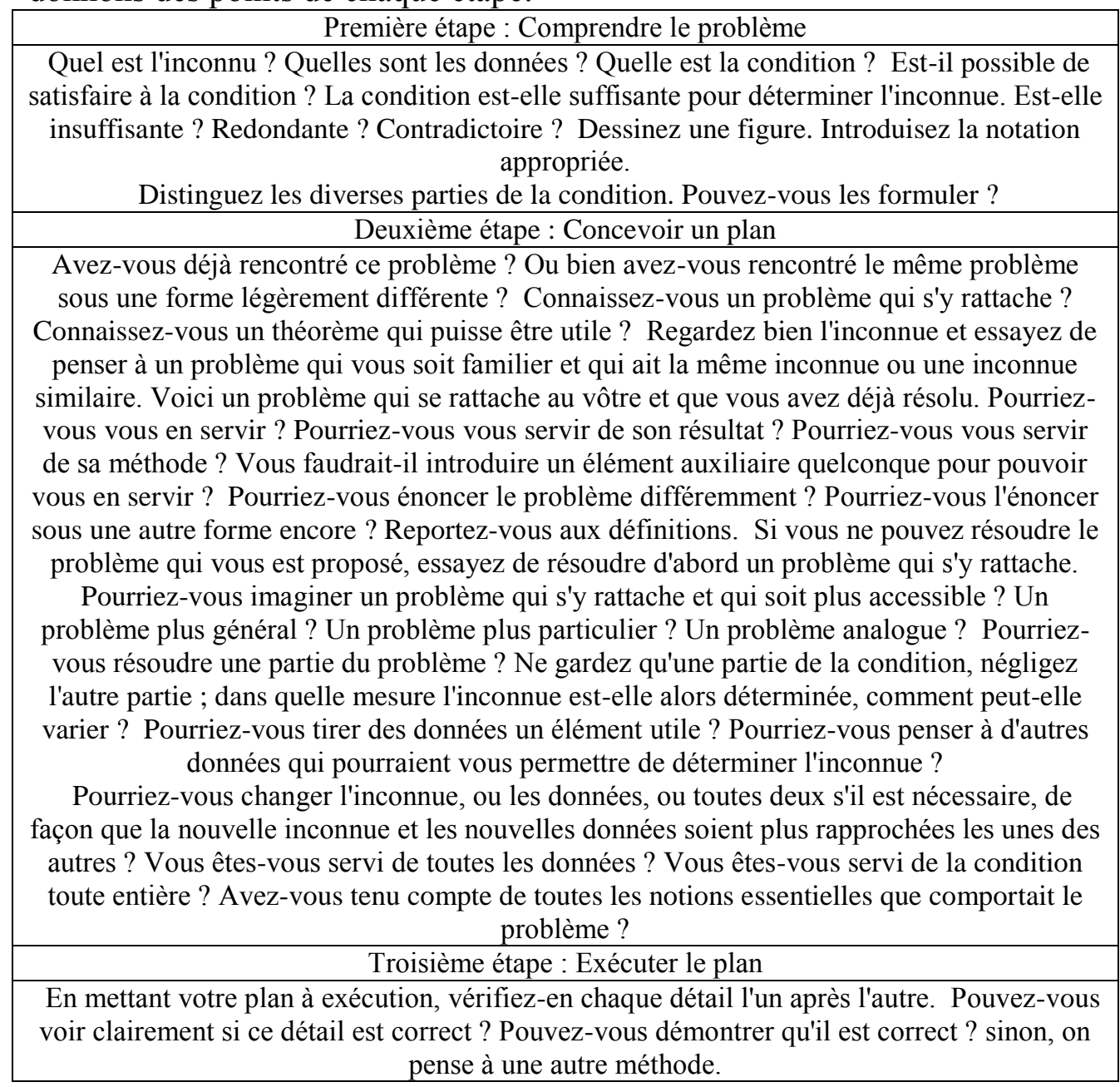


Quatrième étape : Vérifier les résultats

Pouvez-vous vérifier le résultat? Pouvez-vous vérifier le raisonnement ? Pouvez-vous obtenir le résultat différemment ? Pouvez-vous le voir d'un coup d'œil ? Pouvez-vous vous servir du résultat ou de la méthode pour quelque autre problème?

\section{Activités}

\section{Activité 1}

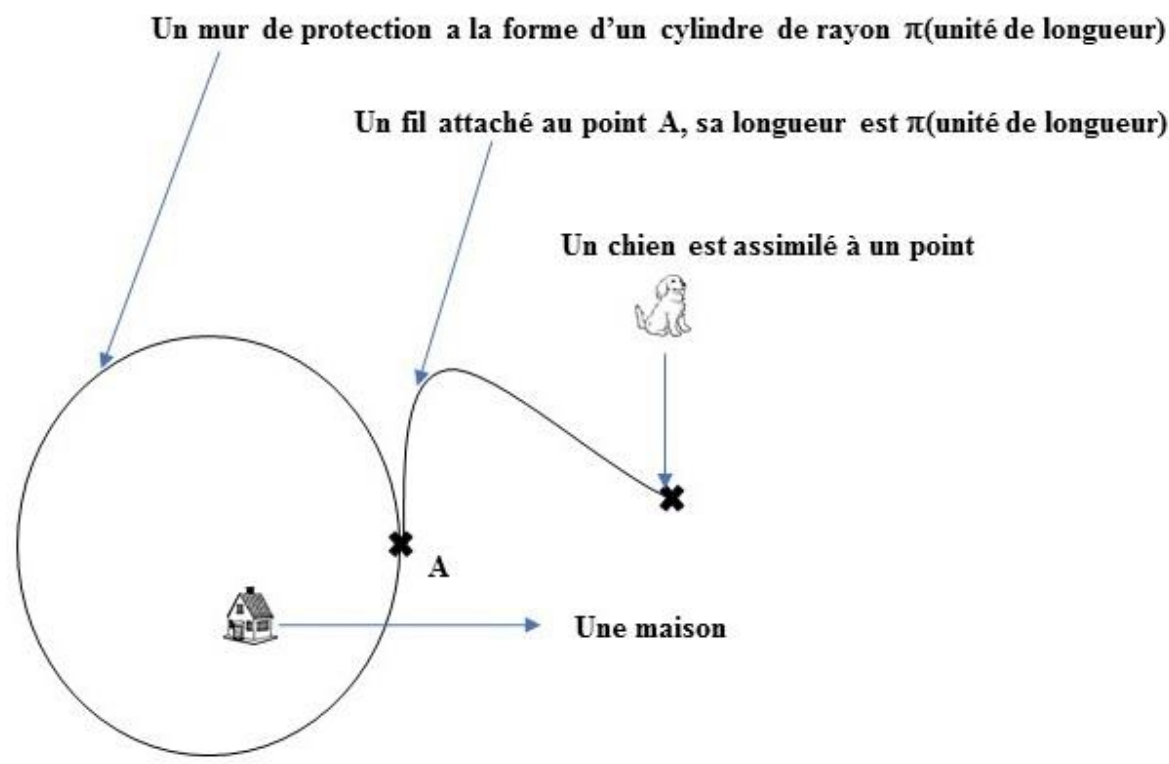

Trouver la frontière de la surface balayée par le chien.

\section{Solution}

Après avoir posé cette activité au professeur stagiaires nous avons constaté qu'en générale les stagiaires n’ont pas des idées pour traiter ce problème.

Nous avons pu prévoir l'allure de la frontière en utilisant une boite de fromage cylindrique et un fil de longueur la moitié de la circonférence et un crayon.

Nous avons attaché le fil d'un côté en un point de la boite et avec le crayon de l'autre côté. Ensuite nous avons posé le tout sur un papier et nous avons tracé la frontière avec le fil tendu.

Finalement nous avons pu traiter un problème facile en remplaçant le mur cylindrique par un mur de forme parallélépipède rectangle de base carrée. A l'aide du logiciel GeoGebra tracer l'allure de la frontière : (le fil attaché au point $\mathrm{E}$ ). 


\begin{tabular}{|l|l|l|l|l|l|l|l|}
\hline $\begin{array}{l}\mathrm{N}^{\circ} \text { de } \\
\text { 1'étape : }\end{array}$ & 1 & 2 & 3 & 4 & 5 & 6 & 7 \\
\hline tracer & $\begin{array}{l}\text { Le } \\
\text { carré } \\
\mathrm{ABCD}\end{array}$ & $\begin{array}{l}\text { Les } \\
\text { droites } \\
(\mathrm{AD}) \\
\text { et } \\
(\mathrm{AB})\end{array}$ & $\begin{array}{l}\text { Le } \\
\text { cercle } \\
\mathrm{C}(\mathrm{E}, 4)\end{array}$ & $\begin{array}{l}\text { F et G } \\
\text { Points } \\
\text { d'intersection } \\
\text { de C(E,4) et } \\
(\mathrm{AD})\end{array}$ & $\begin{array}{l}\text { Arc(AG) } \\
\text { de centre E } \\
\text { Arc(GJ) } \\
\text { de centre A } \\
\text { Arc(JK) } \\
\text { de centre B }\end{array}$ & $\begin{array}{l}\text { La frontière } \\
\text { est } \\
\text { symétrique } \\
\text { Par rapport } \\
\text { à (KE) }\end{array}$ & $\begin{array}{l}\text { ON } \\
\text { colore } \\
\text { La } \\
\text { Frontière } \\
\text { par } \\
\text { le rouge }\end{array}$ \\
\hline
\end{tabular}

Tableau 1. Etape de représentation sur GeoGebra

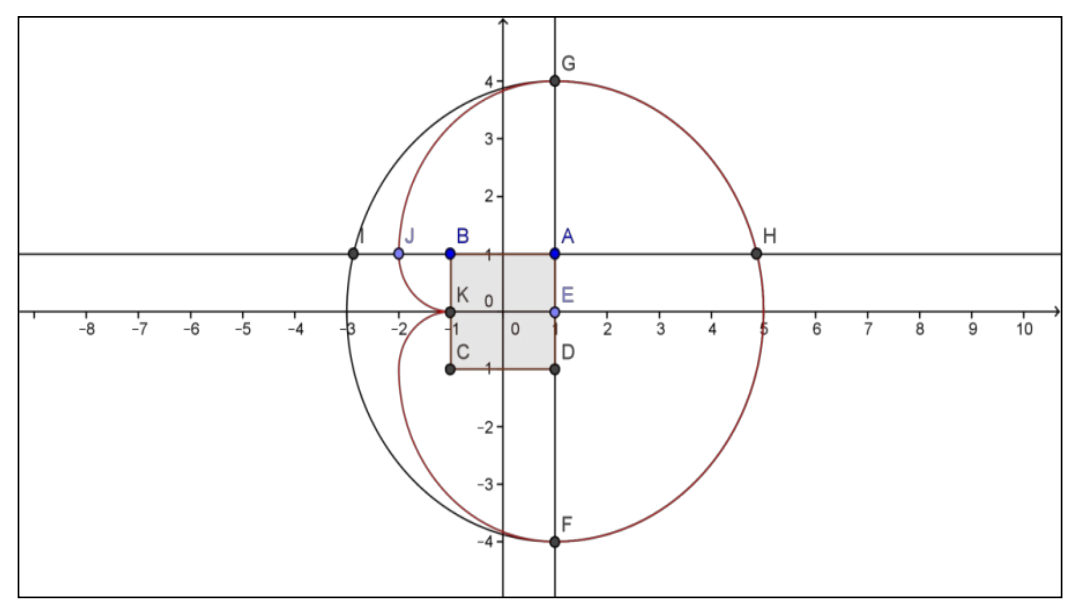

Figure 2. L'allure de la frontière par GeoGebra

- Représentation graphique à l'aide du logiciel Geogebra lorsque le mur a la forme d'un cylindre.

\begin{tabular}{|c|c|c|c|c|c|c|c|}
\hline $\mathrm{N}^{\circ} 1^{\prime}$ étape : & 1 & 2 & 3 & 4 & 5 & 6 & 7 \\
\hline tracer & $\begin{array}{l}\text { Le } \\
\text { Cercle } \\
\mathrm{C}(\mathrm{O}, 1) \\
\text { Et Le } \\
\text { Point } \\
\mathrm{B}(1,0)\end{array}$ & $\begin{array}{l}\text { Le } \\
\text { Curseur } \\
\text { a avec } \\
\text { MIN=0 } \\
\text { MAX=Pi }\end{array}$ & $\begin{array}{l}\text { Le } \\
\text { Point } \\
B^{\prime} \text { tel que } \\
L^{\prime} \text { arc } \\
\left(\mathrm{BB}^{\prime}\right)=\mathrm{a}\end{array}$ & $\begin{array}{l}{\left[\mathrm{B}^{\prime} \mathrm{C}\right]} \\
\text { Tangente } \\
\mathrm{Au} C(\mathrm{O}, 1) \\
\text { au point } \mathrm{B}^{\prime} \\
\text { avec } \\
\mathrm{B}^{\prime} \mathrm{C}=\mathrm{P}_{1-\mathrm{a}}\end{array}$ & $\begin{array}{l}\mathrm{C}^{\prime} \\
\text { symétrique } \\
\text { De C par } \\
\text { Rapport à } \\
\mathrm{X}^{\prime} \mathrm{OX}\end{array}$ & $\begin{array}{l}\text { Lieus du } \\
\text { Points } \\
\mathrm{C} \text { et } \mathrm{C}^{\prime} \\
\text { Qui } \\
\text { dépend } \\
\text { Du } \\
\text { curseur a }\end{array}$ & $\begin{array}{l}\text { Demi- } \\
\text { cercle } \\
\text { (EF) } \\
\text { De centre } \\
\text { B et de } \\
\text { Rayon } \\
\mathrm{Pi}\end{array}$ \\
\hline
\end{tabular}

Tableau 2. Etapes de représentation avec le mur cylindrique 


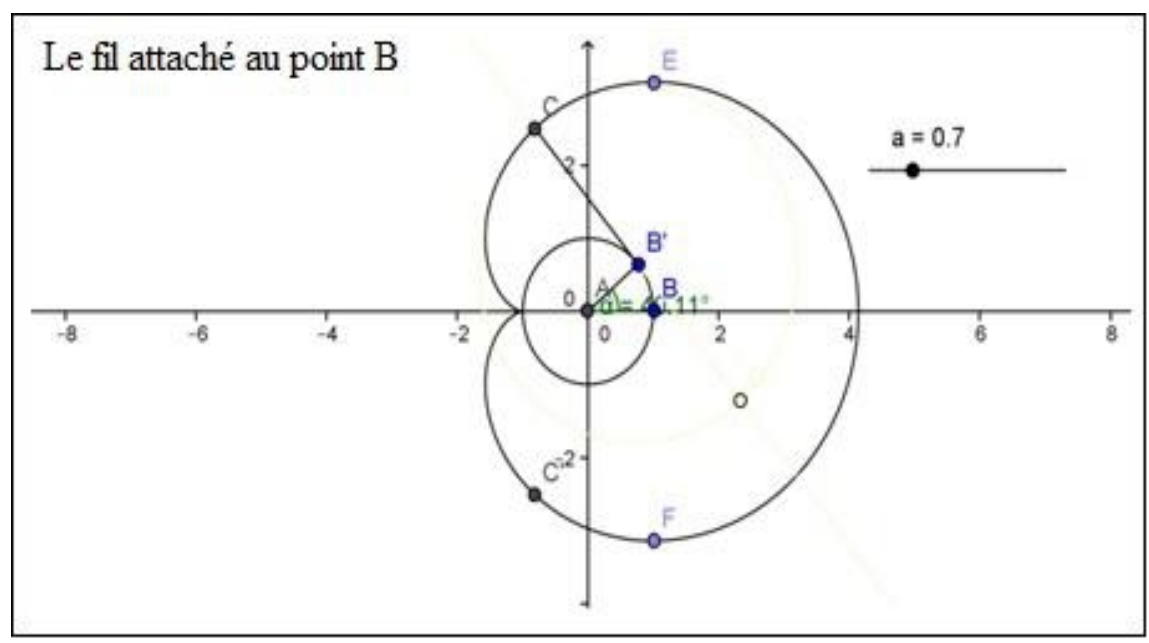

Figure 3. L'allure de représentation avec le mur cylindrique

\section{Solution Mathématique du Problème}

Remarque : Les notations se sont les notations de la Figure 2.

Nous avons fait recours à la géométrie analytique, On considère un repère $(0, \vec{i}, \vec{\jmath})$ d'origine un point $O$ du plan, on construit un cercle de centre $\mathrm{O}$ et de rayon l'unité (Voir la Figure 2). Le fil est attaché au point $\mathrm{B}$, sa longueur est $\mathrm{Pi}$, un point $\mathrm{C}$ de coordonnées $(\mathrm{x}, \mathrm{y})$ est un point de la frontière, il est atteint lorsque le fil est tendu; le demi-cercle d'équation $(x-1)^{2}+$ $y^{2}=$ Pi avec $x>1$ est évident que c'est une partie de la frontière. Le nombre Pi ou $\pi$ est le rapport constant de la circonférence d'un cercle à son diamètre de valeur approchée 3,141592653589793 à $10^{-15}$ prés. Lorsque $x$ est inférieur à un, la longueur du fil est la somme de la longueur de l'arc $\widehat{B B^{\prime}}$ et la longueur $B^{\prime} C$, On a donc: $\mathrm{B}^{\prime} \mathrm{C}=\mathrm{Pi}-\mathrm{a}$ avec a est la mesure de l'angle $\left[\widehat{B O B}^{\prime}\right]$ en radian.

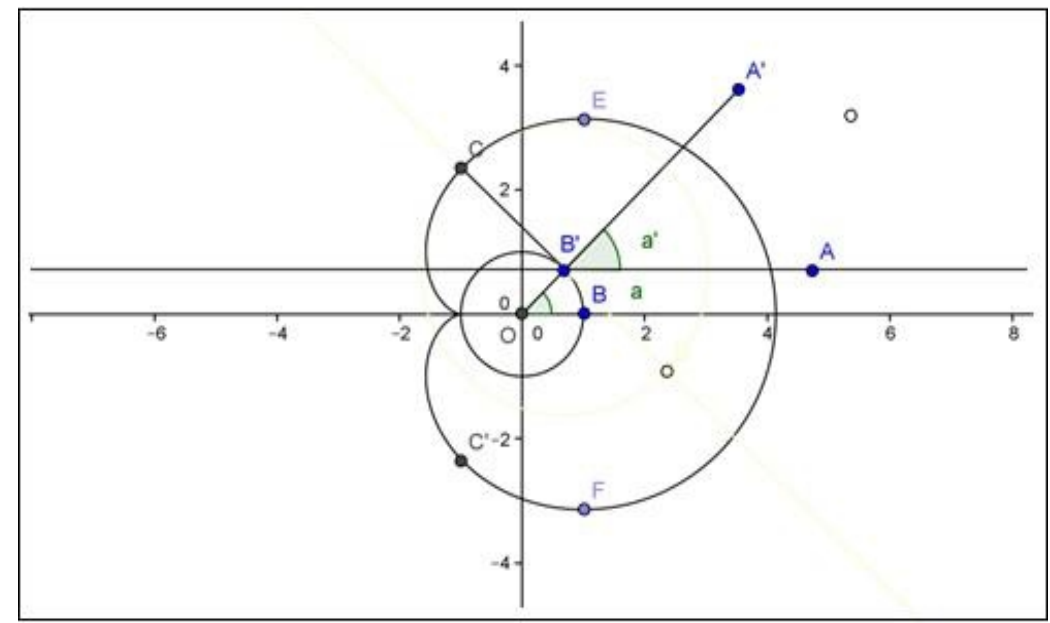

Figure 3. Figure géométrique avec différentes mesures 
On remarque que: $\mathrm{a}^{\prime}=\mathrm{a}$ (deux angles alternes-internes sont égaux)

On a: $\overrightarrow{\mathrm{OC}}=\overrightarrow{\mathrm{OB}^{\prime}}+\overrightarrow{\mathrm{B}^{\prime} \mathrm{C}}$

$$
\begin{aligned}
& =\cos (a) \cdot \vec{\imath}+\sin (a) \cdot \vec{\jmath}+ \\
& \quad(\pi-a)\left[\cos \left(a+\frac{\pi}{2}\right) \cdot \vec{\imath}+\sin \left(a+\frac{\pi}{2}\right) \cdot \vec{\jmath}\right] \\
& =[\cos (a)-(\pi-a) \sin (a)] \cdot \vec{\imath}+[\sin (a)+(\pi-a) \cos (a)] \cdot \vec{\jmath}
\end{aligned}
$$

Donc l'équation paramétrique de la frontière est :

$$
\left\{\begin{array}{l}
x(a)=\cos (a)-(\pi-a) \sin (a) \\
y(a)=\sin (a)+(\pi-a) \cos (a)
\end{array}\right.
$$

Cette courbe paramétrique est facile à étudier et à représenter, mais nous l'avons représenté à l'aide du logiciel Maple (Maple est un logiciel de calcul formel interactif basé sur les expressions mathématiques) par les étapes suivantes:

$>$ restart:

$>$ with(plots):

$>$ with(plottools):

$>\operatorname{disply}\left(\operatorname{disply}\left(\operatorname{arc}\left([1,0], \mathrm{Pi},-\frac{\pi}{2} \cdot \frac{\pi}{2}\right)\right), \operatorname{plot}([\cos (\mathrm{a})-(\mathrm{Pi}-\mathrm{a}) \cdot \sin (\mathrm{a}), \sin (\mathrm{a})+\right.$ $(P i-a) \cdot \cos (a), a=0 . . \pi]), \operatorname{plot}([\cos (a)-(P i-a) \cdot \sin (a),-\sin (a)-(P i-a) \cdot \cos (a), a=0 . . \pi]))$

\section{$\underline{\text { Résultat }}$}

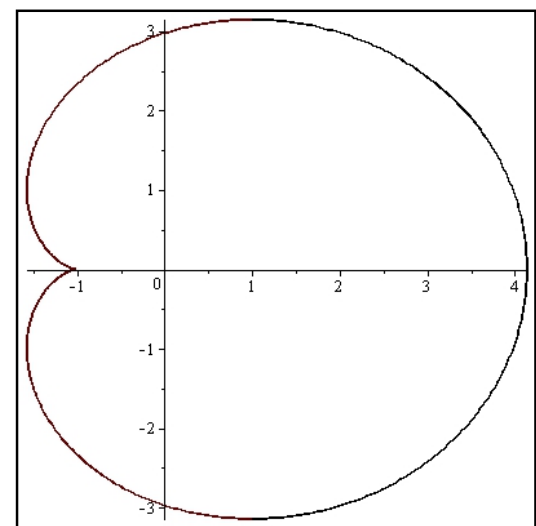

Figure 4. Figure représentative en utilisant Maple

\section{Activité 2}

J'ai deux fois l'âge que vous aviez quand j'avais l'âge que vous avez. Quand vous aurez l'âge que j'ai, nous aurions à nous deux 63 ans. Quel est mon âge ? quel est le vôtre ? Peut-on dire que c'est un père qui parle avec son enfant?

\section{$\underline{\text { Solution }}$}

Les stagiaires trouvent que c'est un problème passionnant ; avec un tel exercice on casse la routine et on aime la séance des mathématiques. Le 
problème contient des verbes à l'imparfait, des verbes au présent et d'autre au future, qui pose des difficultés chez les stagiaires. Voilà une méthode pour résoudre le problème. Par modélisation des données, on aura le tableau suivant:

\begin{tabular}{|c|c|c|c|}
\hline & Au passé & Au présent & Au futur \\
\hline Age du parleur & $\mathrm{y}$ & $2 \mathrm{x}$ & $63-2 \mathrm{x}$ \\
\hline Age de l'écouteur & $\mathrm{x}$ & $\mathrm{y}$ & $2 \mathrm{x}$ \\
\hline
\end{tabular}

Tableau 3. Modélisation chronologique des âges

A partir du tableau, on déduit que $2 x-y=y-x$ et $63-2 x-2 x=2 x-y$ d'où la solution: $\mathrm{y}=21$ et $\mathrm{x}=14$.

Interprétation de la solution: l'âge du parleur est 21 ans et l'âge de l'écouteur 14 ans.

La différence de l'âge est 7 ans, quand l'enfant avait un an, le père avait huit ans, impossible.

\section{Activité 3}

$\mathrm{ABC}$ est un triangle rectangle en $\mathrm{A}$, Déterminer l'ensemble des points $\mathrm{M}$ tel que la droite (AM) est une bissectrice de l'angle [ $\widehat{\mathrm{BMC}}$ ].

\section{Solution}

- Première Méthode: On utilise la géométrie analytique

On considère un repère orthonormé d'origine $\mathrm{A}$ tel que $\mathrm{B}$ a pour coordonnées $(1,0)$ et le point $\mathrm{C}$ a pour coordonnées $(0, \mathrm{c})$. C'est un nombre réel positif, On note les coordonnées de M par $(\mathrm{r}, \mathrm{s})$.

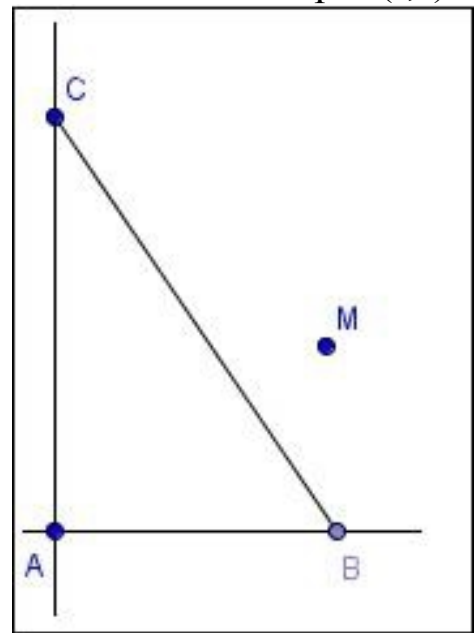

Figure 5. Représentation géométrique du problème 


\section{Les étapes à Suivre}

$>$ On écrit l'équation de la droite $(\mathrm{AM}): \mathrm{ry}-\mathrm{sx}=0$.

$>$ On écrit l'équation de la droite $(\mathrm{BM}): \mathrm{sx}+(1-\mathrm{r}) \mathrm{y}-\mathrm{s}=0$.

$>$ On écrit l'équation de la droite $(\mathrm{CM}):(\mathrm{c}-\mathrm{s}) \mathrm{x}+\mathrm{ry}-\mathrm{cr}=0$.

$>$ (AM) est bissectrice de l'angle(CMB) si est seulement si le point B' symétrique du point $\mathrm{B}$ par rapport à la droite (AM), appartient à la droite (CM).

$>$ Pour trouver les coordonnées de B', on écrit que les droites (BB') et $(\mathrm{AM})$ sont perpendiculaires et que le milieu de [BB'] appartient à (AM).

$>$ On remplace les coordonnées de B' dans l'équation de (CM), On trouve: $\operatorname{cr} 3+\mathrm{crs} 2-\mathrm{cr} 2+\mathrm{cs} 2-\mathrm{r} 2 \mathrm{~s}-\mathrm{s} 3=0$, donc 1'ensemble des points cherché est la courbe d'équation: cx3+cxy2-cx $2+c y 2-x 2 y-y 3=0$. $\left(C^{\prime}\right)$ est une courbe qu'on peut tracer en passant aux coordonnées polaires ou en utilisant des logiciels comme Maple ou GeoGebra.

- Deuxième Méthode : Sans calcul, en utilisant GeoGebra

\section{Les étapes à Suivre}

$>$ Ouvrir une fenêtre du Logiciel GeoGebra.

$>$ Construire un repère orthonormé d'origine $\mathrm{A}$ tel que $\mathrm{B}$ a pour coordonnées $(1,0)$ et le point $\mathrm{C}$ a pour coordonnées $(0, \mathrm{c})$. c' est un nombre réel positif.

$>$ On crée un curseur qui représente un paramètre $\alpha$ qui varie entre 0 et 900.

$>$ On construit une droite (d) qui fait un angle de mesure $\alpha$ avec[AB).

$>$ On construit B' symétrique de $\mathrm{B}$ par rapport à $(\mathrm{d})$.

$>$ La droite (B'C) coupe (d) en un point $\mathrm{M}$, la droite (AM) est une bissectrice de l'angle (BMC), donc $\mathrm{M}$ est un point de l'ensemble cherché. Réciproquement, si M est un point de l'ensemble cherché, la droite (AM) est une bissectrice de l'angle (BMC), donc B' symétrique de $\mathrm{B}$ par rapport à $(\mathrm{AM})$ est un élément de $(\mathrm{CM})$ et la droite (AM) fait un angle de mesure $\alpha$ entre 0 et 900 avec[AB).

$>$ A l'aide de l'outil « lieu du point $M$ quand le curseur varie de 0 à $900 »$ on obtient le lieu du point M, il est coloré en vert. 


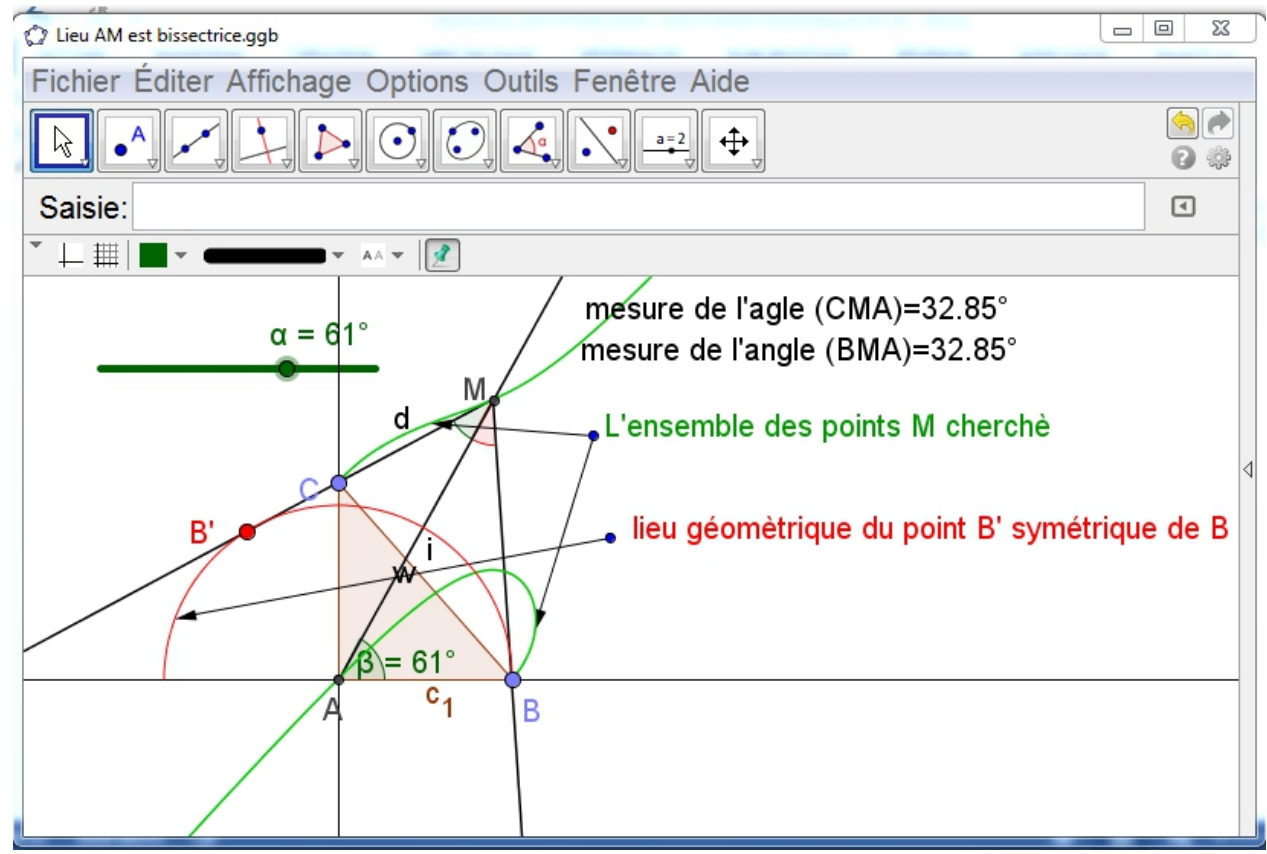

Figure 5. Résultat de constriction par GeoGebra

$>$ On fixe le point $\mathrm{C}$ et on fait varier $\alpha$, pour observer les variations de l'ensemble des points $\mathrm{M}$.

$>$ Lorsque $\mathrm{AB}=\mathrm{AC}, \mathrm{l}$ 'ensemble des points $\mathrm{M}$ cherché est un demicercle de diamètre $\mathrm{BC}$ union les points de la droite d'équation $« y=x »$, que l'on ne peut pas voir dans la figure car le point B' est confondu avec le point $\mathrm{C}$.

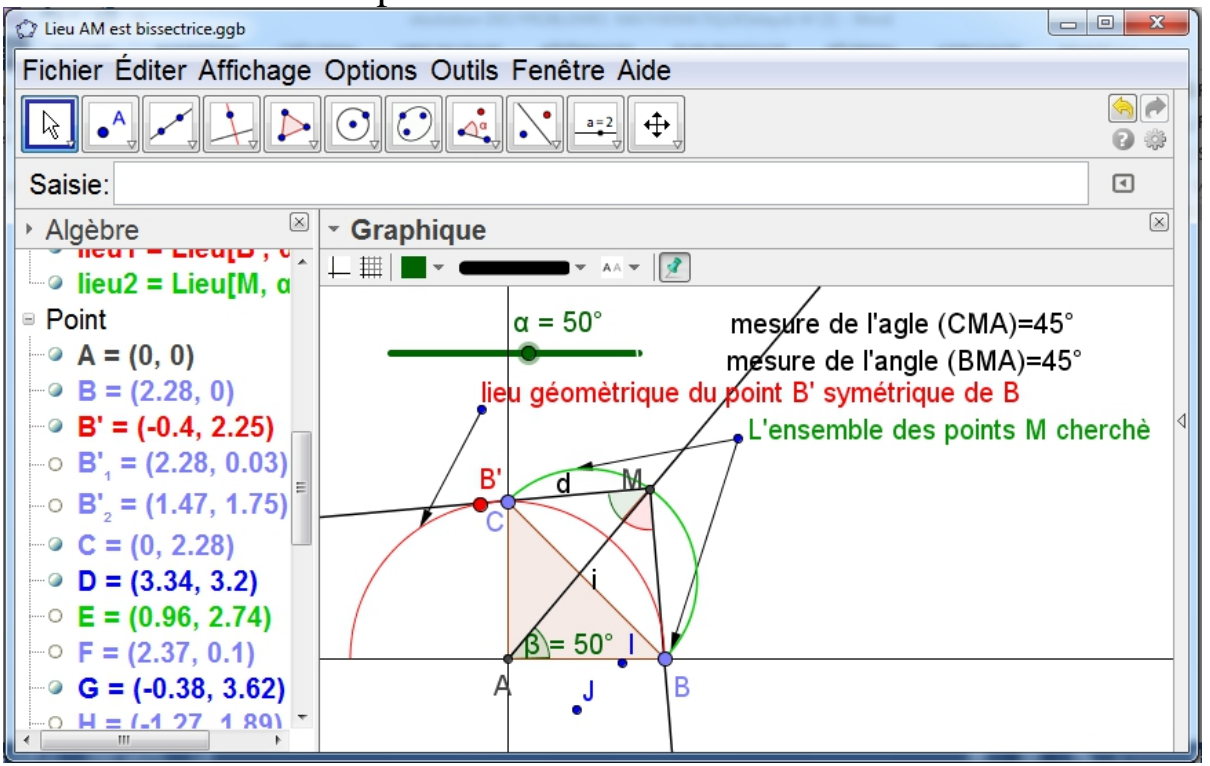

Figure 6. Le lieu géométrique du point $\mathrm{M}$ 


\section{Résolution des Problèmes Ouverts}

\section{Qu'est-ce Qu'un Problème Ouvert ?}

Gilbert ARSAC et Michel MANTE l'IREM de Lyon propose la définition suivante :

«Un problème ouvert est un problème qui possède les caractéristiques suivantes:

- l'énoncé est court.

- L'énoncé n'induit ni la méthode, ni la solution (pas de questions intermédiaires ni de questions du type "montrer que"). En aucun cas, cette solution ne doit se réduire à l'utilisation ou l'application immédiate des derniers résultats présentés en cours.

- Le problème se trouve dans un domaine conceptuel avec lequel les élèves ont assez de familiarité. Ainsi, peuvent-ils prendre facilement "possession" de la situation et s'engager dans des essais, des conjectures, des projets de résolution, des contre-exemples.»

\section{Le Problème Ouvert, Pourquoi ?}

Le problème ouvert permet de proposer à l'élève une activité comparable à celle du mathématicien confronté à des problèmes qu'il n'a pas appris à résoudre. Problème ouvert et situation-problème pourraient ainsi renvoyer à deux aspects du travail du mathématicien:

- Dans le cas du problème ouvert, il s'agit d'abord de chercher une solution originale, personnelle, avec les moyens du bord, mais la solution générale n'est pas à portée de main.

- Dans le cas de la situation-problème, il s'agit, à partir d'un problème particulier, d'élaborer une connaissance (notion, procédure, ...) de portée plus générale et destinée à être institutionnalisée, reconnue socialement, maîtrisée par chacun.

\section{Le Problème Ouvert et Objectifs}

Le problème ouvert permet de mettre l'accent sur des objectifs spécifiques, d'ordre méthodologique, déjà évoqués plus haut. Il exige en effet de l'apprenant, la mise en œuvre des méthodes et de compétences peu travaillées par ailleurs : essayer, organiser sa démarche, mettre en œuvre une solution originale, en mesurer l'efficacité, argumenter à propos de sa solution ou de celle d'un autre, ...

\section{Le Problème Ouvert Comme Outil de Différentiation Pédagogiques}

Le problème ouvert offre une occasion de prendre en compte les compétences de chaque l'apprenant en classe et même de valoriser les différences intellectuelles entre l'apprenant. En effet, si l'énoncé est le même 
pour tous les apprenants, les solutions peuvent être diverses, plus ou moins rapides, en utilisant des connaissances et des stratégies variées.

\section{$\underline{\text { Activité } 1}$}

\section{Solution}

$$
100 \text { ! se termine par combien de zéros? }
$$

Des stagiaires ont remarqué : pour avoir un zéro, on doit multiplier le nombre 5 par un nombre pair, des stagiaires ont trouvé 20 zéros, d'autres sont fiers, ils ont trouvé 22 zéros; d'autres n'ont pas remarqué que le nombre 25 peut produire deux zéros en le multipliant par 4 ; la bonne réponse est 24; en effet:

On suppose qu'on a développé l'entier 100 ! en produit de nombres premiers, on aura: $100 !=2^{\mathrm{a}} \times 5^{\mathrm{b}} \times 3^{\mathrm{c}} \times \ldots . \ldots .$. , il y'a autant de multiple de deux que les multiples de 5 dans le produit.

$2 \times 3 \times 4 \times 5 \times \ldots \ldots \ldots . \ldots 100$, donc 100 ! se termine par c zéros,

Il y'a 20 multiples de 5 qui sont 5 x 1, 5 x 2, ., 5 x 20 et quatre multiples de 25 donc il y'a 24 zéros.

En utilisant le logiciel Maple, on trouve 100 ! = 9332621544394415268169923885626670049071596826438162146859296 3895217599993229915608941463976156518286253697920827223758251 185210916864000000000000000000000000

\section{Activité 2} satisfaite?

Peut' on effacer l'un des symboles + ou - pour que l'égalité (E) soit

$$
\pm 1 \pm 2 \pm 3 \pm 4 \pm \ldots \ldots \ldots \ldots \ldots \ldots \ldots \pm 100=2009
$$
satisfaite?

Peut' on effacer l'un des symboles + ou - pour que l'égalité (F) soit

$$
\pm 1 \pm 2 \pm 3 \pm 4 \pm \cdots \ldots \ldots \ldots \ldots \ldots \ldots \pm 100=2010
$$

\section{Solution}

Nous avons constaté que les stagiaires pensent toujours positivement, ils ne doutent pas que la réponse peut être négative, des stagiaires arrivent à trouver 2008, d'autre 2010, Nous nous sommes demandé comment peut-on faire pour ajouter 1 ou retrancher 1. En réalité, il est impossible de réaliser l'égalité (E), en effet : supposons que l'égalité (E) est réalisable, on note par A la somme des nombres positifs et par B la somme des nombres négatifs, on a donc : $\mathrm{A}+\mathrm{B}=2009$ et $\mathrm{A}-\mathrm{B}=1+2+3+\ldots \ldots \ldots+100=5050$ d'où $(\mathrm{A}+\mathrm{B})$ $+(\mathrm{A}-\mathrm{B})=2009+5050$ donc $2 \mathrm{~A}=7059$ impossible, $($ pair $=$ impair $)$.

L'égalité (F) est possible, on laisse au lecteur de trouver une solution. 


\section{Activité 3}

Lors d'une soirée, cinq couples se rencontrent, chaque personne étant donc avec son conjoint. Je constate, moi qui fait partie de ce groupe de 10 personnes que certains n'ont pas donné la main à tout le monde. Je demande alors à chacun : à combien de personnes as-tu donnée la main ? j'obtiens alors 9 réponses différentes. Il est bien entendu que toute personne ne se donne pas la main et ne donne pas la main à son conjoint à combien de personnes mon conjoint a-t-il donné la main ?

\section{Solution}

La plupart des stagiaires ne sont pas encore mariés(es), cette activité les rend joyeux, un climat agréable règne dans la classe, mais ils n'arrivent pas à trouver l'idée pour résoudre le problème. Toute personne ne se donne pas la main et ne donne pas la main à son conjoint, donc au maximum une personne donne la main à 9 personnes. Comme il y a 9 réponses différentes, les réponses sont : $0,1,2, \ldots, 9$. Je note par $\mathrm{P}$ le parleur et par $\mathrm{Pi}$ la personne qui a salué i personnes, $(\mathrm{i}=0,1,2, \ldots . ., 9)$; P8 a salué toutes les personnes sauf P0, donc P8 et P0 forment un couple, P7 a salué toutes les personnes sauf P0et P1(P1 a salué P8) donc P7 et P1 forment un couple, par analogie $\mathrm{P} 6$ et $\mathrm{P} 2$ forment un couple et P5 et P3 forment un couple donc P4 et P forment un couple ; conclusion : le conjoint du parleur a salué 4 personnes

\section{Activité 4}

Dans un plan, on considère 6 points tels que 3 quelconques ne sont pas alignés. Chaque segment joignant deux de ses points est colorié, (au hasard), soit en vert, soit en rouge. On considère ensuite tous les triangles ayant trois de ces points comme sommets.

Est-il toujours possible de trouver un triangle dont les trois cotés sont de la même couleur ?

\section{Solution}

On note par $\mathrm{A}, \mathrm{B}, \mathrm{C}, \mathrm{D}, \mathrm{E}$ et $\mathrm{F}$ les six points, on considère les segments $[\mathrm{AB}],[\mathrm{AC}],[\mathrm{AD}],[\mathrm{AE}],[\mathrm{AF}]$, on colore ces segments au hasard, dans tous les cas il y'aura toujours trois segments qui ont même couleur, pour fixer les idées, on prend par exemple [AB], [AC], [AD] ont même couleur par exemple rouge, 


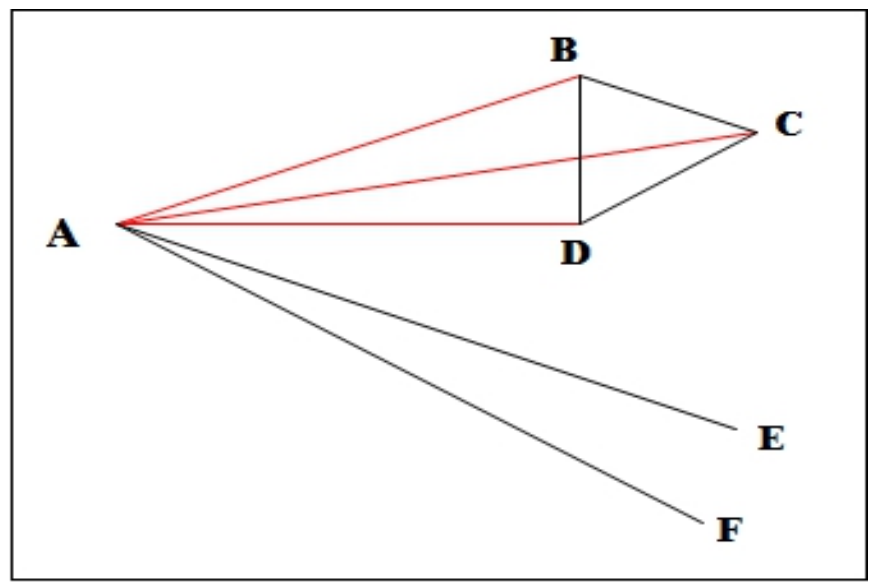

Figure 7. Figure géométrique représentative

Supposons qu'il existe un cas où les côtés de tout triangle n'ont pas même couleur donc:

$[B C]$ est vert sinon les côtés du triangle $\mathrm{ABC}$ ont même couleur [CD] est vert sinon les côtés du triangle ACD ont même couleur [BD] est vert sinon les côtés du triangle ABD ont même couleur

Mais alors $\mathrm{BCD}$ est un triangle dont les côtés ont même couleur, absurde, donc il est toujours possible de trouver un triangle dont les trois cotés sont de la même couleur.

\section{Conclusion}

Les mathématiques est une discipline que nous trouvons partout, depuis le début de notre parcours scolaire jusqu'à notre vie de tous les jours. Nous sommes face elle tout le temps et dans différentes situations.

Elle nous enseigne des méthodes de raisonnement et des méthodologies de travail basées sur des stratégies bien spécifiques pour arriver au résultat recherché.

Dans notre modeste travail, nous avons essayé de montrer comment peut-on mettre en œuvre des stratégies de résolution de problèmes en travaillant sur des exemples des problèmes «non routiniers » qui stimulent et poussent l'apprenant à réfléchir, relever le défi et s'engager intellectuellement dans le processus Enseignement/Apprentissage.

Une méthode souple et adaptable à des situations nouvelles de la vie quotidienne ou professionnelle.

Nous espérons que ce travail servira de plat forme pour des recherches plus avancées sur le sujet, d'être un outil pour la formation des enseignants des mathématiques et qu'il soit une ouverture à la diversité des démarches de résolution de problèmes visant et l'apprentissage des contenus mathématiques et le développement des heuristiques de résolution. 


\section{References:}

1. APMEP (1987). Association des professeurs de mathématiques de l'enseignement public. Elem-math IX, Brochure $\mathrm{n}^{\circ}$ 64. Situations problèmes.

2. Arsac, G., Germain, G., \& Mante, M. (1988). Problème ouvert et situation-problème.

3. Arsac, G., \& Mante M. (2007). Les pratiques du problème ouvert. SCEREN CRDP de Lyon.

4. Arsac, G., Chapiron, G., Colonna, A., Germain, G., Guichard, Y., \& Mante, M. (1992). Initiation au raisonnement déductif au collège, Presses Universitaires de Lyon.

5. Arsac, G., Germain, G., Mante, M., \& Pichot, D. (1985a). La pratique du problème ouvert, IREM de Lyon.

6. Arsac, G., Germain, G., Mante, M., \& Pichot, D. (1985b). Varions notre enseignement avec les problèmes ouverts, IREM de Lyon.

7. Artigue, M. \& Houdement, C. (2007). Problem solving in France: didactic and curricular perspectives, ZDM, 39(5-6), 365-382.

8. Becker, J. \& Shimada, S. (1997), The open-ended approach: a new proposal for teaching mathematics. Reston, VA : National Council of Teachers of Mathematics.

9. Bouvier, B. (1986). Didactique des Mathématiques : le lire et le faire, CEDIC.

10. Brousseau, G. (1998). Théorie des situations didactiques, Grenoble : La pensée sauvage.

11. Crespo, S. (2003). Learning to pose mathematical problems: Exploring changes in preservice teachers' practices, Educational Studies in Mathematics, 52, 243-270.

12. Cunningham, R. (2004). Problem posing: an opportunity for increasing student responsibility, Mathematics and Computer Education, 38, 83-89.

13. Directives pédagogiques général pour l'enseignement des mathématiques dans l'enseignement secondaire collégial (2009). Ministère de l'éducation, Maroc.

14. Ermel (de 1991 à 1999). Apprentissages numériques et résolution de problèmes : CP, CE1, CE2, CM1 et CM2, Paris, HATIER.

15. Gilbert Arsac \& Michel Mante (2007). Les pratiques du problème ouvert, IREM de Lyon, P20.

16. Gompertz, A. (2016). Les problèmes ouverts, une piste de différenciation pédagogique? (CM1).

17. Houdement, C. (1998). Le choix des problèmes pour la résolution de problèmes. Grand N, 63, 59-77. 
18. Kosyvas, G. (2013). Pratiques pédagogiques de problèmes ouverts dans un collège expérimental à Athènes. Repères IREM, Numéro 91.

19. Polya, G. (1957). How to solve it: a new aspect of mathematical method. Garden City N.Y Doubleday. Rosati LA.

20. Robert, A. (1998). Outil d'analyse des contenus mathématiques à enseigner au lycée et à l'université. Recherches en Didactique des Mathématiques. Vol 18 (2) pp 139-190.

21. Robert, A. \& Rogalski, M. (2004). Problèmes d'introduction et autres problèmes de recherché au lycée, Repère IREM, 54, 77-103.

22. Silver, E.A. (1994). On Mathematical Problem Posing, For the Learning of Mathematics, 14(1), 19-28. 